OPEN ACCESS

Edited by: lan Dunn,

University of Oklahoma Health Sciences Center, United States

Reviewed by: Tim J. Kruser, University of Wisconsin System, United States Jiang Wu,

Soochow University, China

*Correspondence: Ye Gong

gong_ye@fudan.edu.cn Lingyang Hua hua.ling.yang@163.com

${ }^{t}$ These authors have contributed equally to this work

Specialty section: This article was submitted to Neuro-Oncology and Neurosurgical Oncology, a section of the journa Frontiers in Oncology

Received: 26 July 2021 Accepted: 30 September 2021 Published: 29 October 2021

Citation:

Deng J, Sun S, Chen J, Wang $D$, Cheng $H$, Chen $H$, Xie $Q$, Hua $L$ and

Gong Y (2021) TERT Alterations

Predict Tumor Progression in De Novo High-Grade Meningiomas Following Adjuvant Radiotherapy. Front. Oncol. 11:747592. doi: 10.3389/fonc.2021.747592

\section{TERT Alterations Predict Tumor Progression in De Novo High-Grade Meningiomas Following Adjuvant Radiotherapy}

\author{
Jiaojiao Deng ${ }^{1,2,3 \dagger}$, Shuchen Sun ${ }^{1,2,3 \dagger}$, Jiawei Chen ${ }^{1,2,3 \dagger}$, Daijun Wang ${ }^{1,2,3}$, Haixia Cheng ${ }^{4}$, \\ Hong Chen ${ }^{4}$, Qing Xie ${ }^{1,2,3}$, Lingyang Hua ${ }^{1,2,3 *}$ and Ye Gong ${ }^{1,2,3,5 *}$ \\ 1 Department of Neurosurgery, Huashan Hospital, Fudan University, Shanghai, China, 2 Institute of Neurosurgery, Fudan \\ University, Shanghai, China, ${ }^{3}$ Shanghai Key Laboratory of Brain Function Restoration and Neural Regeneration, Fudan \\ University, Shanghai, China, ${ }^{4}$ Department of Neuropathology, Huashan Hospital, Fudan University, Shanghai, China, \\ ${ }^{5}$ Department of Critical Care Medicine, Huashan Hospital, Fudan University, Shanghai, China
}

Background: Adjuvant radiotherapy (RT) is one of the most commonly used treatments for de novo high-grade meningiomas (HGMs) after surgery, but genetic determinants of clinical benefit are poorly characterized.

Objective: We describe efforts to integrate clinical genomics to discover predictive biomarkers that would inform adjuvant treatment decisions in de novo HGMs.

Methods: We undertook a retrospective analysis of 37 patients with de novo HGMs following RT. Clinical hybrid capture-based sequencing assay covering 184 genes was performed in all cases. Associations between tumor clinical/genomic characteristics and $\mathrm{RT}$ response were assessed. Overall survival (OS) and progression-free survival (PFS) curves were plotted using the Kaplan-Meier method.

Results: Among the 172 HGMs from a single institution, 42 cases (37 WHO grade 2 meningiomas and five $\mathrm{WHO}$ grade 3 meningiomas) were identified as de novo HGMs following RT. Only TERT mutations [62.5\% C228T; 25\% C250T; 12.5\% copy number amplification (CN amp.)] were significantly associated with tumor progression after postoperative RT (adjusted $p=0.003$ ). Potential different somatic interactions between TERT and other tested genes were not identified. Furthermore, TERT alterations (TERT-alt) were the predictor of tumor progression (Fisher's exact tests, $p=0.003$ ) and were associated with decreased PFS (log-rank test, $p=0.0114$ ) in de novo HGMs after RT.

Conclusion: Our findings suggest that TERT-alt is associated with tumor progression and poor outcome of newly diagnosed HGM patients after postoperative RT.

Keywords: high-grade meningiomas, adjuvant radiotherapy, de novo, TERT alterations, prognosis 


\section{INTRODUCTION}

Meningiomas are the most frequent tumors of the central nervous system and are generally benign $(1,2)$. The World Health Organization (WHO) defines three grades predictive of the risk of recurrence (3). High-grade meningiomas (HGMs) (WHO grades 2-3) are rare but aggressive tumors with considerably poorer prognosis than WHO grade 1 meningiomas $(4,5)$. The 5-year progression-free survival (PFS-5) and overall survival (OS) rate for HGM patients are $8 \%-68 \%$ and $35 \%-91 \%$, respectively (4-8).

Patients with WHO grade 1 meningiomas are traditionally managed in follow-up with surveillance imaging (9). However, a standardized treatment approach to HGMs after resection has not been established (4). The benefit to survival outcomes of HGMs with adjuvant radiotherapy (RT) post-surgical resection remains unclear. Retrospective series on adjuvant RT after gross total resection led to differing results $(4,10,11)$.

Meningiomas have a diverse genetic background that varies with biologic behavior (12). Alterations in the tumor suppressor gene NF2 were the first discovered genetic etiology of meningiomas $(13,14)$. In NF2 wild-type meningiomas, mutations in TRAF7, KLF4, AKT1, and SMO were noted (1517). In addition, several mutations have been described with potential prognostic implications in $\operatorname{HGMs}(12,14,18)$. Data published recently have also shown that activating TERT promoter mutations, frequent inactivation of $B A P 1$, deletions of CDKN2A/ $B$, and mutations in $D M D$ are frequent in meningiomas with malignant histological progression (18-21). These data suggest that convergent gene-expression programs may underlie HGMs, which could be leveraged to develop prognostic biomarkers.

Our previous work found that patients with de novo anaplastic meningiomas benefit from adjuvant RT after surgery $(5,22)$. However, the molecular factors associated with RT efficacy in de novo HGMs are largely unknown. In the present study, we describe efforts to integrate clinical genomics of 37 cases from 173 HGMs to address this issue.

\section{METHODS}

\section{Patient Selection}

Patients were identified for study through a review of the clinical records of the Department of Neurosurgery, Huashan Hospital of Fudan University, Shanghai, China. A total of 172 HGMs (Supplementary Table S1) were included following study approval by the Human Subjects Institutional Review Board at Huashan Hospital, Fudan University (KY-2017-09). Clinical characteristics including age, gender, tumor location, extent of surgical resection, and outcome data were collected. In general, adjuvant radiation was recommended to both atypical and anaplastic meningioma patients, regardless of gross total resection (GTR; Simpson grades I-III) or subtotal resection (STR; Simpson grades IV-V). And the final decision was made based on the negotiation with the relatives of patients. The details of postoperative RT were described in our previous work (5). Tumor pathological subtypes were reconfirmed by at least two experienced neuropathologists. Follow-up was conducted routinely according to the guidelines of Huashan Neurosurgical Center. Written informed consent was obtained from all patients involved in our study.

A total of 172 patients with a confirmed diagnosis of HGM who met inclusion criteria (141 WHO grade 2 meningiomas and 31 WHO grade 3 meningiomas; Supplementary Table S1). Out of 172 cases, 87 cases received RT after surgery. Among the 87 patients, $42(48.3 \%)$ were de novo meningiomas, while the remaining 45 patients presented with recurrent meningioma following prior surgical resection. And finally, 37 de novo meningioma samples with adequate quality of DNA concentration were included for further next-generation sequencing.

\section{Next-Generation Sequencing}

Tumor genotyping was performed on formalin-fixed paraffinembedded (FFPE) tumor tissue by next-generation sequencing (NGS) covering 184 genes, including common pathological relevant genes of meningiomas (Supplementary Table S2) (13-21, 23). Five DNA samples were excluded for sequencing due to inadequate quality of concentration. High-throughput sequencing was performed on Illumina miniseq platform by KuoRan Biomedical Technology as previously described (Supplementary Material) (24).

\section{Sanger Sequencing}

The TERT promoter mutations were evaluated using Sanger sequencing. Genomic DNA was obtained from FFPE using the HiPure FFPE DNA Kit (Magen, D3126-03) following polymerase chain reaction-based amplification of the target region (forward primer: GGATTCGCGGGC ACAGAC; reverse primer: CAGCGCTGCCTGAAA CTC; details on PCR conditions are available upon request).

\section{Statistical Tests}

The specific details of statistical tests are included in the figure legends. A two-tailed Fisher's exact test was used to calculate statistical significance between different groups using a $\chi^{2} 2 \times 2$ table. Categorical variables were compared with the Fisher's exact tests, and continuous variables with the independentsamples Student's $t$-test (data with normal distribution) or Mann-Whitney U-test (data with skewed distribution). Continuous data were expressed as the mean \pm standard deviation (SD). Overall survival (OS) and progression-free survival (PFS) curves were plotted using the Kaplan-Meier method. Statistical analysis was performed using Statistical Package for Social Sciences (SPSS, Version 20.0, Chicago, IL, USA). Data were considered to be significant when $p<0.05$.

\section{RESULTS}

\section{Prevalence of Somatic Alterations in $\mathbf{3 7}$ De Novo High-Grade Meningiomas}

We identified 172 patients with a confirmed diagnosis of HGM who met inclusion criteria (141 WHO grade 2 meningiomas and 
31 WHO grade 3 meningiomas; Supplementary Table S1). Out of 172 cases, 87 cases received RT after surgery, including external beam radiation therapy (EBRT), stereotactic radiosurgery (SRS), and cyber knife (CK). Among the 87 patients, $42(48.3 \%)$ were de novo meningiomas while the remaining 45 patients presented with recurrent meningioma following prior surgical resection. Five of the 42 (11.9\%) meningiomas were excluded for sequencing due to inadequate quality of DNA concentration. Thus, tumor genotyping covering 184 genes was performed on the 37 de novo meningioma cases (Supplementary Tables S2, S3). In total of the 37 cases, 23 males (62\%) and 14 females (38\%) with a median age of 45 years (range: $34-73$ ) harboring 31 atypical (83.8\%), five anaplastic (13.5\%), and one atypical/chordoid coexisting $(2.7 \%)$ meningiomas were included. Tumors were located at the convexity $(\mathrm{n}=17,45.9 \%)$, the falx/parasagittal $(\mathrm{n}=13,35.1 \%)$, the skull base $(n=5,13.5 \%)$, or in other locations $(n=2,5.5 \%)$. Among these patients, 28 (75.7\%) were treated with EBRT, six $(16.2 \%)$ were treated with SRS, and three cases were treated with CK (8.1\%) (Figure 1).

In order to identify tumor mutations associated with efficacy of postoperative RT, we determined the association between recurrent mutations and tumor progression in the cohort of 37 patients (Figure 1). Of the 37 de novo HGMs following RT, 19 cases $(51.4 \%)$ had tumor progression. Progression individuals was defined as tumor regrowth within the radiation field based on the criterion of Response Assessment in Neuro-Oncology Working Group (RANO) radiologic criteria for meningiomas
(25). Part of the genomic mutational landscape of 37 patients with de novo meningioma is displayed in Figure 2. Nine mutations met our predetermined recurrence frequency threshold of $>20 \%$ (Figure 2 and Supplementary Table S2). Consistent with previous studies, high mutational rates of NF2 $(\mathrm{n}=22 ; 59 \%)$ were discovered in this cohort. Additional common pathological relevant genes of meningiomas, including AKT1 ( $\mathrm{n}=3 ; 8 \%), C D K N 2 A(\mathrm{n}=2 ; 5 \%), S M O(\mathrm{n}=0 ; 0 \%), S U F U$ $(\mathrm{n}=0 ; 0 \%), P O L R 2 A(\mathrm{n}=6 ; 16 \%), \operatorname{TRAF7}(\mathrm{n}=1 ; 3 \%)$, and SMARCB1 ( $\mathrm{n}=2 ; 5 \%)$, were observed as well (Supplementary Table S2). Besides, the most frequently altered genes including $\operatorname{ATRX}(\mathrm{n}=13 ; 35 \%)$, ARID1A ( $\mathrm{n}=11 ; 30 \%), \operatorname{ATM}(\mathrm{n}=11 ; 30 \%)$, NF1 ( $=11 ; 30 \%)$, ROS1 $(\mathrm{n}=10 ; 27 \%)$, KDM6A $(\mathrm{n}=9 ; 24 \%)$, FAT1 $(\mathrm{n}=8 ; 22 \%)$, and TERT $(\mathrm{n}$ $=8 ; 22 \%$ ) were observed (Figure 2).

\section{TERT Mutations Predict Tumor Progression of De Novo High-Grade Meningiomas Following Radiotherapy}

Strikingly, only TERT mutations were significantly associated with tumor progression $(\mathrm{n}=8$, adjusted $p=0.031)$, and all these mutations were present in tumors that progressed after RT (Figure 3A). Of the TERT mutant cases, $87.5 \%$ (7/8) presented with TERT promoter mutations (62.5\% C228T variant and $25 \%$ C250T variant; Supplementary Figure S1), and 12.5\% (1/8) harbored copy number amplification (CN amp.). As many tumor driver genes are co-occurring or show strong exclusiveness in their mutation pattern, we next explored the potential different somatic interactions in the cohort. None of the gene mutations

Work-flow:

172 high-grade meningiomas<smiles>C1C2CC12</smiles>

87 received postoperative $R T$

1

37 de novo high-grade meningiomas:

WHO grade 2 ( $n=32)$, WHO grade $3(n=5)$

$\downarrow$

184 targeted gene panel sequencing

Representative driver genes of meningiomas for sequencing

$\begin{array}{lllll}\text { NF2 } & \text { TRAF7 } & \text { KLF4 } & \text { AKT1 } & \text { SMO } \\ \text { CDK2NA/B } & \text { BAP1 } & \text { TERT } & \text { DMD } & \text { ect. }\end{array}$

FIGURE 1 | Study design. 


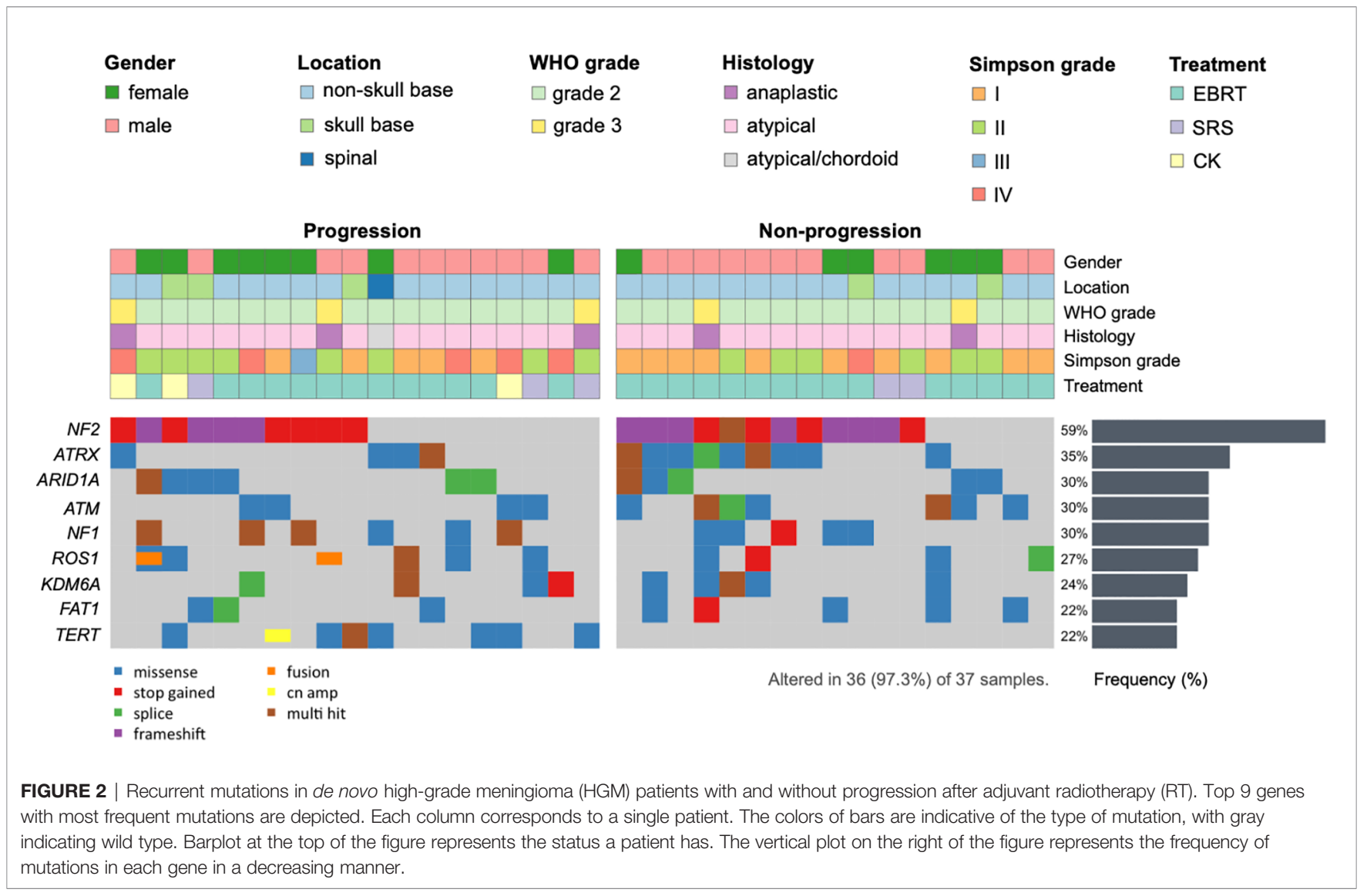

A

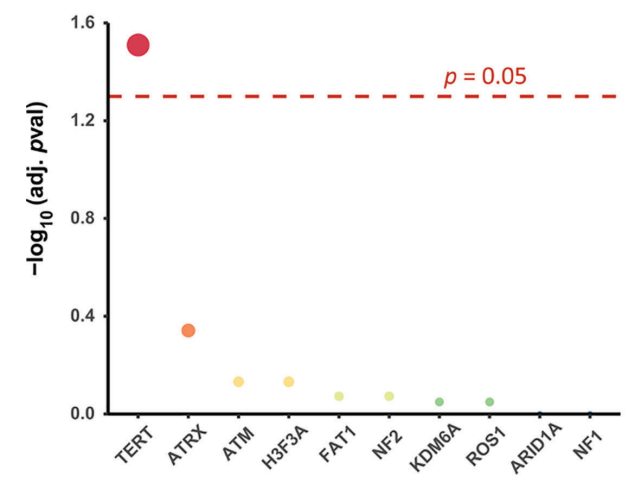

B

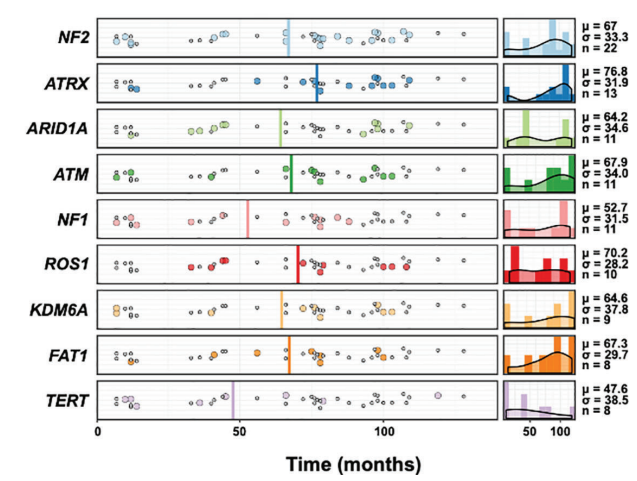

FIGURE 3 | TERT mutations predict tumor progression of de novo high-grade meningiomas (HGMs) following radiotherapy (RT). (A) Association of recurrent mutations with tumor progression. Fisher's test was utilized to detect differentially mutated genes on top 9 most frequent mutation genes between two cohorts (progression vs. non-progression). The point size in dotplot corresponds to the -log10(adj. $p$-val) value, together with the red color indicates the higher -log10(adj. $p$ val) value, and blue indicates the lower value. Horizontal dash line marked the $p$-value 0.05 . (B) Timing of tumor progression. Shown is the time to progression (colored dots) or last progression-free scan (gray dots) for top 9 most frequent mutation genes in months. The average progression time was depicted in vertical line. The average time to recurrence of TERT mutant meningiomas was less than other tumors (Wilcoxon rank sum test with continuity correction, $\mathrm{W}=491, p=0.0864$ ). Density plot of each subgroup's progression is shown on the right, along with the mean $(\mu)$, standard deviation $(\sigma)$, and number of progression (n).

presents co-occurring or mutually exclusive in HGM cases (Supplementary Figure S2). Thus, TERT mutations appear to be the dominant cause of tumor progression among mutations in this cohort. Furthermore, we found that TERT mutation status
( $p=0.003)$, as well as extent of resection (EOR) $(p<0.001)$, was significantly associated with tumor progression by Fisher's exact tests (Table 1). Neither tumor location $(p=1.00)$ nor WHO grade $(p=1.00)$ predicted tumor progression after postoperative 
TABLE 1 | Analysis of progression factors in de novo HGM patients after RT.

\begin{tabular}{|c|c|c|c|}
\hline Feature & Progression & Non-progression & $p$-value \\
\hline Patients, n (\%) & $19(51.35)$ & $18(48.65)$ & \\
\hline \multicolumn{4}{|l|}{ Age, n (\%) } \\
\hline$<65$ & $13(68.42)$ & $14(77.78)$ & 0.71 \\
\hline$\geq 65$ & $6(31.58)$ & $4(22.22)$ & \\
\hline \multicolumn{4}{|l|}{ Gender, n (\%) } \\
\hline Male & $11(57.89)$ & $12(66.67)$ & 0.74 \\
\hline Female & $8(42.11)$ & $6(33.33)$ & \\
\hline \multicolumn{4}{|l|}{ Location, n (\%) } \\
\hline Skull base & $3(15.79)$ & $2(11.11)$ & 1.00 \\
\hline Non-skull base & $16(84.21)$ & $16(88.89)$ & \\
\hline \multicolumn{4}{|l|}{ WHO grade, n (\%) } \\
\hline 2 & $16(84.21)$ & $16(88.89)$ & 1.00 \\
\hline 3 & $3(15.79)$ & $2(11.11)$ & \\
\hline \multicolumn{4}{|l|}{ EOR, n (\%) } \\
\hline GTR & 14 (73.68) & $1(5.56)$ & $<0.001^{* *}$ \\
\hline STR & $5(26.32)$ & $17(94.44)$ & \\
\hline \multicolumn{4}{|l|}{ TERT status, n (\%) } \\
\hline TERT (+) & $8(42.11)$ & $0(0)$ & $0.003^{*}$ \\
\hline TERT (-) & $11(57.89)$ & $18(100)$ & \\
\hline
\end{tabular}

GTR, gross total resection; HGM, high-grade meningioma; $R T$, radiotherapy; STR, subtotal resection; EOR, extent of resection.

${ }^{*} p<0.05$ and ${ }^{* *} p<0.001$ considered statistically significant.

RT. After adjustment for multiple comparisons, these associations were not significant. Importantly, TERT mutation cases were more likely to have unfavorable time to recurrence (TTR) over the entire cohort, with a mean observation time of 47.6 months (Figure 3B). However, no significant differences in the average time to recurrence between TERT mutant and wildtype cases were observed (Wilcoxon rank sum test with continuity correction $\mathrm{W}=491, p=0.0864)$.

Meningioma with TERT alterations, regardless of WHO grades or pathological subtypes, had a highly significant risk of recurrence (26). To exclude the disruption to RT efficacy might be caused by TERT-related malignant biological behavior, we thus performed analysis on newly diagnosed HGMs depending on TERT alterations only in progression group. Of the 19 cases in the progression group, mitotic index (ki-67\%) depending on TERT alterations did not show any significantly difference (unpaired t-test, $p=0.051$; Supplementary Figure S3A).
Additionally, TERT alterations of de novo HGMs had no predictive effect on tumor recurrence in progression group following postoperative RT ( $p=0.074$ with log-rank test; Supplementary Figure S3B).

\section{TERT Mutations Were Associated With Decreased Progression-Free Survival and Overall Survival in De Novo High-Grade Meningiomas After Radiotherapy}

With these findings, we next analyzed the effect of TERT mutation status on PFS in patients with de novo HGMs after RT. The primary endpoint of PFS, defined as time from surgery to date of progression, was assessed on the basis of progression of meningioma after initial surgery on imaging follow-up. The median PFS of 75 months (range: 7-109 months) was observed for the entire cohort. Subgroup analysis revealed a median PFS of 25 months (range: 10-79 months) in TERT mutant group and 77 months (range: 7-109 months) in TERT wild-type group. TERT mutants were significantly associated with decreased PFS in de novo HGM cases that underwent postoperative RT ( $p=0.0114$ with log-rank test; Figure 4A).

The relationship between TERT mutations and OS was analyzed using the log-rank test (Figure $\mathbf{4 B}$ ). The median OS of 84 months (range: 13-123 months) was observed for the entire cohort. Subgroup analysis revealed a median OS of 66 months (range: 13-123 months) in TERT mutant group and 85 months (range: 24-109 months) in TERT wild-type group. Patients with de novo HGMs after RT harboring TERT mutations had worse OS $(p=0.0562$; Figure 4B).

\section{DISCUSSION}

In this retrospective study, we investigated genetic predictors that might inform the potential progressive risk in de novo HGMs after postoperative RT. We found TERT-alt in HGMs to be a significant predictor of tumor progression compared to TERT wild-type cases. Although a merely descriptive finding, our
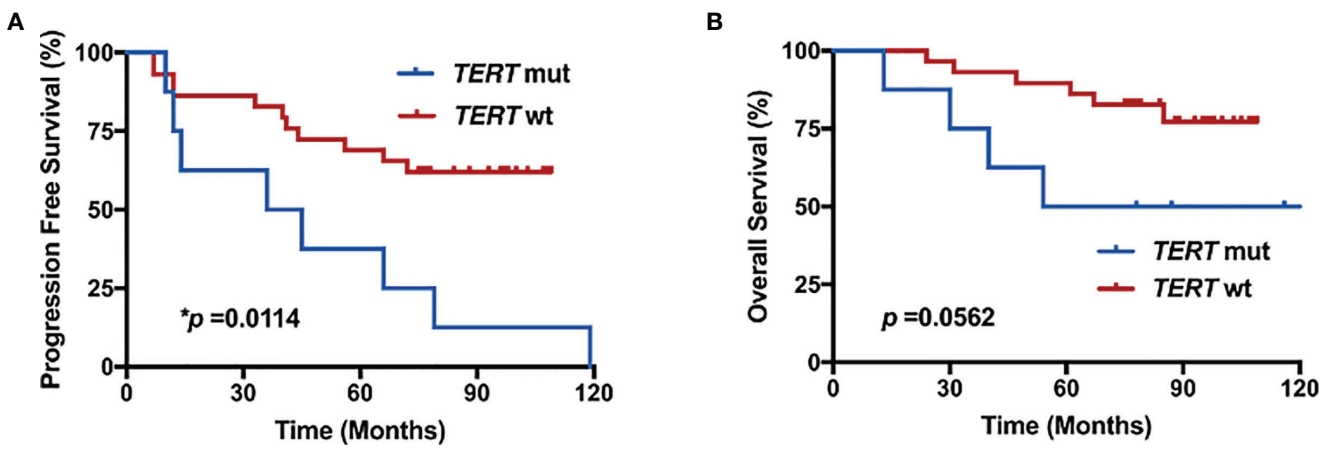

FIGURE 4 | TERT mutations were associated with decreased progression-free survival (PFS) and overall survival (OS) in de novo high-grade meningiomas (HGMs) after radiotherapy (RT). Kaplan-Meier curves for (A) PFS and (B) OS in de novo HGMs with or without TERT mutation following RT. 
data have potential implications for the clinical management of patients with TERT-alt de novo HGMs.

HGMs are rare but aggressive tumors with considerably poorer prognosis than lower-grade meningiomas. Adjuvant RT is the only nonsurgical standard of care treatment option for these tumors (9). However, radiotherapeutic options for meningioma are diverse, and there are no randomized trials to identify individuals who are more likely to benefit from adjuvant RT. Indeed, until recently, the evidence supporting postoperative RT for meningioma, especially HGMs, was largely limited. Moreover, no molecular prognostic markers have yet been established for new diagnosed HGMs following RT. To our knowledge, this study is the first to investigate predictive biomarkers that would inform tumor progression in de novo HGMs after postoperative RT.

Leveraging the next-generation sequencing techniques led to advances in description of the mutational landscape of meningiomas (15-17). In line with the previous findings, NF2mutant meningiomas represent the largest percentage (59\%) of cases in our study $(14,15)$. In large-scale genomic studies of meningioma, HGMs were in some studies exclusively related to pathogenic variants in NF2, associated with mutations in the TERT promoter (27). In our study, high mutational rates of ATRX, ARID1A, ATM, NF1, ROS1, KDM6A, FAT1, and TERT were observed, indicating that these frequently altered genes might play a role in HGMs. Of note, other common pathological relevant genes of meningiomas, including $A K T 1$ ( $\mathrm{n}=3 ; 8 \%$ ), CDKN2A ( $\mathrm{n}=2 ; 5 \%), S M O(\mathrm{n}=0 ; 0 \%), \operatorname{SUFU}(\mathrm{n}=0 ; 0 \%)$, POLR2A $(\mathrm{n}=6 ; 16 \%)$, TRAF7 $(\mathrm{n}=1 ; 3 \%)$, and SMARCB1 $(\mathrm{n}=2$; $5 \%)$, were detected as well. However, we did not observe previously described mutational rates of some of these genes due to limited cases.

Among the 184 sequenced genes, only TERT alterations were significantly associated with tumor progression $(n=8$, adjusted $p=0.031$ ), and all these mutations were present in tumors that progressed after RT. TERT-alt comprise, but are not limited to, promoter mutations, gene translocations, and DNA amplifications (28). We found $87.5 \%$ (7/8) of the TERT mutant cases presented with TERT promoter mutations $(62.5 \%$ C228T variant and $25 \% \mathrm{C} 250 \mathrm{~T}$ variant). As reported, the most common alterations occur in specific "hotspots" of the promoter (TERTp) region known as C228T and C250T (29). These C>T transition mutations lead to maintenance of the telomere length, as binding of E-twenty six (ETS)-transcription factors is involved in the upregulation of TERT expression $(28,30)$.

TERT alterations, specifically TERT promoter mutations, have been identified in a subset of HGMs with progression from low-grade meningioma (31-34). In the present study, we found that TERT-alt was associated with decreased PFS and OS in de novo HGMs after RT. Besides, our data have shown decreased time to progression among TERT-alt de novo HGMs as well. TERT gene is transcriptionally inactive in most nonneoplastic cells (28). TERT gene alterations (TERT-alt) may enforce cell immortalization by counteracting telomere shortening, thus promoting growth (28). Several studies have provided evidence that TERT-alt mutations are associated with rapid recurrence and malignant progression in meningioma (26, $31,35)$. In addition, TERTp meningiomas have been found to have a worse PFS and OS, though not many cases have been reported (18). Activating TERT gene mutations in the upstream promoter allows overexpression of this enzyme and is responsible for immortalization of tumor cells in many cancers (12). Thus, we have excluded that the disruption to RT efficacy might be caused by TERT-related malignant biological behavior in this study. As results, the TERT-alt status and progressive variables did not show any relevance in progression group.

The Simpson grade of EOR has long been used to guide clinical expectations after resection of meningiomas (9); our results support the relevance of EOR in recurrence of HGMs as well $(p<0.001)$. The literature widely recognizes the role that EOR plays in determining HGM prognosis $(36,37)$. However, Cox regression analysis failed to identify any factor with significant association with the progression of de novo HGMs following RT. Thus, a larger cohort or multicenter clinical trial is needed to investigate the effect of RT in this subgroup.

In summary, our data identified TERT alterations, especially TERTp mutation, are associated with tumor progression and poor outcome of newly diagnosed HGM patients after postoperative RT. Several limitations of this study warrant consideration. Firstly, our findings on a discovery series were not substantiated by any independent validation series due to the limited available samples. Prospective studies are clearly needed to validate TERT-alt status of radiation response in de novo HGMs. Another important limitation in this study is its observational nature, which could have led to selection bias. It would be useful to repeat these analyses in cohorts from other institutions in the future.

\section{CONCLUSION}

In summary, examining a cohort of de novo HGMs following adjuvant RT, we find TERT alteration to be strongly associated with tumor progression and poor outcome of HGM patients included in this study.

\section{DATA AVAILABILITY STATEMENT}

The datasets presented in this study can be found in online repositories. The names of the repository/repositories and accession number(s) can be found below: NCBI [accession: PRJNA753598].

\section{ETHICS STATEMENT}

The studies involving human participants were reviewed and approved by the Human Subjects Institutional Review Board at Huashan Hospital, Fudan University. The patients/participants provided their written informed consent to participate in this study. Written informed consent was obtained from the individuals for the publication of any potentially identifiable images or data included in this article. 


\section{AUTHOR CONTRIBUTIONS}

JD, LH, and YG, study design. JD, SS, JC, DW, and QX, clinical data. JD, article preparation. $\mathrm{HaC}$ and $\mathrm{HoC}$, tumor pathological subtypes reconfirmed and Sanger sequencing. All authors contributed to the article and approved the submitted version.

\section{FUNDING}

This study was supported by grants from the National Key R\&D Program of China (2018YFC1312600 and 2018YFC1312604 to YG) and the National Natural Science Foundation of China (82072788 and 81772674 to YG).

\section{REFERENCES}

1. Kshettry VR, Ostrom QT, Kruchko C, Ossama A-M, Barnett GH, BarnholtzSloan JS, et al. Descriptive Epidemiology of World Health Organization Grades II and III Intracranial Meningiomas in the United States. Neuro Oncol (2015) 17:1166-73. doi: 10.1093/neuonc/nov069

2. Ostrom QT, Gittleman H, Liao P, Rouse C, Chen Y, Dowling J, et al. CBTRUS Statistical Report: Primary Brain and Central Nervous System Tumors Diagnosed in the United States in 2007-2011. Neuro Oncol (2014) null:iv163. doi: 10.1093/neuonc/nou223

3. Louis DN, Perry A, Wesseling P, Brat DJ, Cree IA, Figarella-Branger D, et al. The 2016 World Health Organization Classification of Tumors of the Central Nervous System: A Summary. Acta Neuropathol (2016) 131:803-20. doi: 10.1007/s00401-016-1545-1

4. Unterberger A, Nguyen T, Duong C, Kondajji A, Kulinich D, Yang I, et al. Meta-Analysis of Adjuvant Radiotherapy for Intracranial Atypical and Malignant Meningiomas. J Neurooncol (2021) 152:205-16. doi: 10.1007/ s11060-020-03674-7

5. Wang D, Sun S, Hua L, Deng J, Luan S, Cheng H, et al. Prognostic Model That Predicts Benefits of Adjuvant Radiotherapy in Patients With High Grade Meningioma. Front Oncol (2020) 10:568079. doi: 10.3389/fonc.2020.568079

6. Yang S-Y, Park C-K, Park S-H, Kim DG, Chung YS, Jung H-W, et al. Atypical and Anaplastic Meningiomas: Prognostic Implications of Clinicopathological Features. J Neurol Neurosurg Psychiatry (2008) 79:574-80. doi: 10.1136/ jnnp.2007.121582

7. Dziuk TW, Woo S, Butler EB, Thornby J, Grossman R, Dennis WS, et al. Malignant Meningioma: An Indication for Initial Aggressive Surgery and Adjuvant Radiotherapy. J Neurooncol (1998) 37:177-88. doi: 10.1023/ A:1005853720926

8. Choi Y, Lim DoH, Jo K, Nam D-H, Seol HJ, Lee J-I, et al. Efficacy of Postoperative Radiotherapy for High Grade Meningiomas. J Neurooncol (2014) 119:405-12. doi: 10.1007/s11060-014-1507-1

9. Goldbrunner R, Minniti G, Preusser M, Jenkinson MD, Sallabanda K, Houdart E, et al. EANO Guidelines for the Diagnosis and Treatment of Meningiomas. Lancet Oncol (2016) 17:e383-91. doi: 10.1016/S1470-2045(16)30321-7

10. Mair R, Morris K, Scott I, Carroll TA. Radiotherapy for Atypical Meningiomas. J Neurosurg (2011) 115:811-9. doi: 10.3171/2011.5.JNS11112

11. Rogers CL, Won M, Vogelbaum MA, Perry A, Ashby LS, Modi JM, et al. HighRisk Meningioma: Initial Outcomes From NRG Oncology/RTOG 0539. Int $J$ Radiat Oncol Biol Phys (2020) 106:790-9. doi: 10.1016/j.jijobp.2019.11.028

12. Bunevicius A, Laws ER, Saudargiene A, Tamasauskas A, Iervasi G, Deltuva V, et al. Common Genetic Variations of Deiodinase Genes and Prognosis of Brain Tumor Patients. Endocrine (2019) 66:563-72. doi: 10.1007/s12020-019-02016-6

13. Yuzawa S, Nishihara H, Tanaka S. Genetic Landscape of Meningioma. Brain Tumor Pathol (2016) 33:237-47. doi: 10.1007/s10014-016-0271-7

14. Birzu C, Peyre M, Sahm F. Molecular Alterations in Meningioma: Prognostic and Therapeutic Perspectives. Curr Opin Oncol (2020) 32:613-22. doi: $10.1097 / \mathrm{CCO} .0000000000000687$

\section{SUPPLEMENTARY MATERIAL}

The Supplementary Material for this article can be found online at: https://www.frontiersin.org/articles/10.3389/fonc.2021. 747592/full\#supplementary-material

Supplementary Figure S1 | Pie chart fraction of TERT alterative subtypes occurring in TERT-alt HGMs.

Supplementary Figure S2 | Heatmap of mutually exclusive or co-occurring set of genes in the mutation pattern of de novo HGMs following RT. Pairwise Fisher's exact tests were performed to detect significant pairs of genes, mutually exclusive or co-occurring set of genes which colored by brown or green can be detected using the somaticInteractions function in R/Bioconductor package 'maftools'.

Supplementary Figure S3 | The TERT-alt status and progressive variables did not show any relevancy in progression group.

15. Clark VE, Erson-Omay EZ, Serin A, Yin J, Cotney J. Genomic Analysis of non-NF2 Meningiomas Reveals Mutations in TRAF7, KLF4, AKT1, and SMO. Science (2013) 339:1077-80. doi: 10.1126/science.1233009

16. Clark VE, Harmanci AS, Bai H, Youngblood MW, Lee TI, Baranoski JF, et al. Recurrent Somatic Mutations in POLR2A Define a Distinct Subset of Meningiomas. Nat Genet (2016) 48:1253-9. doi: 10.1038/ng.3651

17. Delgado-López PD, Cubo-Delgado E, González-Bernal JJ, Martín-Alonso J. A Practical Overview on the Molecular Biology of Meningioma. Curr Neurol Neurosci Rep (2020) 20:62. doi: 10.1007/s11910-020-01084-w

18. Biczok A, Kraus T, Suchorska B, Terpolilli NA, Thorsteinsdottir J, Giese A. TERT Promoter Mutation Is Associated With Worse Prognosis in WHO Grade II and III Meningiomas. J Neurooncol (2018) 139:671-8. doi: 10.1007/ s11060-018-2912-7

19. Shankar GM, Santagata S. BAP1 Mutations in High-Grade Meningioma: Implications for Patient Care. Neuro Oncol (2017) 19:1447-56. doi: 10.1093/ neuonc/nox094

20. Sievers P, Hielscher T, Schrimpf D, Stichel D, Reuss DE, Berghoff AS, et al. CDKN2A/B Homozygous Deletion Is Associated With Early Recurrence in Meningiomas. Acta Neuropathol (2020) 140:409-13. doi: 10.1007/s00401020-02188-w

21. Juratli Tareq A, McCabe D, Nayyar N, Williams EA, Silverman IM, Tummala SS, et al. DMD Genomic Deletions Characterize a Subset of Progressive/ Higher-Grade Meningiomas With Poor Outcome. Acta Neuropathol (2018) 136:779-92. doi: 10.1007/s00401-018-1899-7

22. Zhu H, Bi WL, Aizer A, Hua L, Tian M, Den J, et al. Efficacy of Adjuvant Radiotherapy for Atypical and Anaplastic Meningioma. Cancer Med (2019) 8:13-20. doi: 10.1002/cam4.1531

23. Toland A, Huntoon K, Dahiya SM. Meningioma: A Pathology Perspective. Neurosurgery (2021) 89:11-21. doi: 10.1093/neuros/nyab001

24. Jin L, Shi F, Chun Q, Chen H, Ma Y, Wu S, et al. Artificial Intelligence Neuropathologist for Glioma Classification Using Deep Learning on Hematoxylin and Eosin Stained Slide Images and Molecular Markers. Neuro Oncol (2021) 23:44-52. doi: 10.1093/neuonc/noaa163

25. Huang Raymond Y, Bi WL, Weller M, Kaley T, Blakeley, Dunn I, et al. Proposed Response Assessment and Endpoints for Meningioma Clinical Trials: Report From the Response Assessment in Neuro-Oncology Working Group. Neuro Oncol (2019) 21:26-36. doi: 10.1093/neuonc/noy137

26. Mirian C, Duun-Henriksen AK, Juratli T, Sahm F, Spiegl-Kreinecker S, Peyre $\mathrm{M}$, et al. Poor Prognosis Associated With TERT Gene Alterations in Meningioma Is Independent of the WHO Classification: An Individual Patient Data Meta-Analysis. J Neurol Neurosurg Psychiatry (2020) 91:37887. doi: 10.1136/jnnp-2019-322257

27. Sahm F, Schrimpf D, Stichel D, Jones David TW, Hielscher T, Schefzyk S, et al. DNA Methylation-Based Classification and Grading System for Meningioma: A Multicentre, Retrospective Analysis. Lancet Oncol (2017) 18:682-94. doi: 10.1016/S1470-2045(17)30155-9

28. Barthel Floris $\mathrm{P}$, Wei $\mathrm{W}$, Tang M, Martinez-Ledesma E, Hu X, Amin Samirkumar B, et al. Systematic Analysis of Telomere Length and Somatic 
Alterations in 31 Cancer Types. Nat Genet (2017) 49:349-57. doi: 10.1038/ ng. 3781

29. Yuan X, Larsson C, Xu D. Mechanisms Underlying the Activation of TERT Transcription and Telomerase Activity in Human Cancer: Old Actors and New Players. Oncogene (2019) 38:6172-83. doi: 10.1038/s41388-019-0872-9

30. Horn S, Figl A, Rachakonda PS, Fischer C, Sucker A, Gast A, et al. TERT Promoter Mutations in Familial and Sporadic Melanoma. Science (2013) 339:959-61. doi: 10.1126/science.1230062

31. Sahm F, Schrimpf D, Olar A, Koelsche C, Reuss D, Bissel J, et al. TERT Promoter Mutations and Risk of Recurrence in Meningioma. J Natl Cancer Inst (2016) 108(5). doi: 10.1093/jnci/djv377

32. Koelsche C, Sahm F, Capper D, Reuss D, Sturm D, Jones TW, et al. Distribution of TERT Promoter Mutations in Pediatric and Adult Tumors of the Nervous System. Acta Neuropathol (2013) 126:907-15. doi: 10.1007/s00401-013-1195-5

33. Goutagny Stéphane, Nault JC, Mallet M, Henin D, Rossi JZ, Kalamarides M, et al. High Incidence of Activating TERT Promoter Mutations in Meningiomas Undergoing Malignant Progression. Brain Pathol (2014) 24:184-9. doi: 10.1111/bpa.12110

34. Peyre M, Gauchotte G, Giry M, Froehlich S, Pallud J, Graillon T, et al. De Novo and Secondary Anaplastic Meningiomas: A Study of Clinical and Histomolecular Prognostic Factors. Neuro Oncol (2018) 20:1113-21. doi: 10.1093/neuonc/nox231

35. Spiegl-Kreinecker S, Lötsch D, Neumayer K, Kastler L, Gojo J, Kumar C, et al. TERT Promoter Mutations Are Associated With Poor Prognosis and Cell Immortalization in Meningioma. Neuro Oncol (2018) 20:1584-93. doi: 10.1093/neuonc/noy104
36. Nanda A, Bir SC, Konar S, Maiti T, Kalakoti P, Jacobsohn JA, et al. Outcome of Resection of WHO Grade II Meningioma and Correlation of Pathological and Radiological Predictive Factors for Recurrence. J Clin Neurosci (2016) 31:112-21. doi: 10.1016/j.jocn.2016.02.021

37. Sun Sam Q, Hawasli AH, Huang J, Chicoine MR, Kim AH. An EvidenceBased Treatment Algorithm for the Management of WHO Grade II and III Meningiomas. Neurosurg Focus (2015) 38:E3. doi: 10.3171/2015.1. FOCUS14757

Conflict of Interest: The authors declare that the research was conducted in the absence of any commercial or financial relationships that could be construed as a potential conflict of interest.

Publisher's Note: All claims expressed in this article are solely those of the authors and do not necessarily represent those of their affiliated organizations, or those of the publisher, the editors and the reviewers. Any product that may be evaluated in this article, or claim that may be made by its manufacturer, is not guaranteed or endorsed by the publisher.

Copyright $\odot 2021$ Deng, Sun, Chen, Wang, Cheng, Chen, Xie, Hua and Gong. This is an open-access article distributed under the terms of the Creative Commons Attribution License (CC BY). The use, distribution or reproduction in other forums is permitted, provided the original author(s) and the copyright owner(s) are credited and that the original publication in this journal is cited, in accordance with accepted academic practice. No use, distribution or reproduction is permitted which does not comply with these terms. 